ÉGYPTE monde arabe

\section{Égypte/Monde arabe}

$12 \mid 2015$

Evolution des systèmes médiatiques après les révoltes arabes

\title{
Internet, le «Printemps arabe » et la dévaluation du cyberactivisme arabe
}

\section{Yves Gonzalez-Quijano}

\section{(2) OpenEdition}

\section{Journals}

Édition électronique

URL : https://journals.openedition.org/ema/3400

DOI : 10.4000/ema.3400

ISSN : 2090-7273

Éditeur

CEDEJ - Centre d'études et de documentation économiques juridiques et sociales

Édition imprimée

Date de publication : 25 mars 2015

Pagination : 67-84

ISBN : 9782905838858

ISSN : 1110-5097

Référence électronique

Yves Gonzalez-Quijano, «Internet, le «Printemps arabe » et la dévaluation du cyberactivisme arabe », Égypte/Monde arabe [En ligne], 12 | 2015, mis en ligne le 25 mars 2015, consulté le 07 juillet 2022. URL : http://journals.openedition.org/ema/3400 ; DOI : https://doi.org/10.4000/ema.3400 


\section{RÉSUMÉ / ABSTRACT}

Plus de trois ans après ses débuts, il est temps désormais d'interroger la place d'Internet dans ce qu'il est convenu d'appeler le « Printemps arabe ». Après la vague d'enthousiasme suscitée par la figure du " jeune rebelle arabe » et cette cyber-révolution supposée mettre un terme aux divers régimes autoritaires de la région, il est devenu davantage possible de se livrer à un examen plus critique du rôle politique d'Internet et des réseaux sociaux dans des événements qui ont mis en évidence les limites des mobilisations numériques. Dans certains contextes, l'activisme politique en ligne a non seulement perdu sa virginité politique, mais il a également prouvé qu'il pouvait devenir un facteur perturbateur par rapport à la recherche d'un consensus politique. Mais en dépit des événements qui ont porté atteinte à son crédit, le cyberactivisme arabe conserve, sur le long terme, tout son potentiel pour contribuer aux changements sociaux et politiques dans la région.

\section{MOTS CLÉS :}

Internet, cyberactivisme, blogueurs, nouveaux médias.

More than three years after 2011, the time has come to question the impact of the Internet in the context of the so-called "Arab Spring". After the wave of enthusiasm towards the "young Arab rebel" and the Great Cyber Revolution supposed to put an end to the various authoritarian regimes, it has now become more possible to develop a critical understanding of the use of the Internet and the social media in the political sphere, in which the dynamics of the digital mobilizations have showed their limitations. In various contexts, online activism has not only lost its "political virginity" but has also proved to be an unsettling factor for the search of a political consensus. Nonetheless, the events which eroded the reputation of Arab online activism certainly do not totally obliterate its ability to contribute to political and social changes in the long term.

KEYWORDS:

Internet, Digital Activism, Bloggers, New Media. 
YVES GONZALEZ-QUIJANO est maître de conférences au département d'études arabes de I'Université Lumière Lyon2 et membre du GREMMO, Yves-Gonzalez-Quijano est notamment l'auteur d'Arabités numériques. Le printemps du Web arabe (Actes Sud-Sindbad, 2011).

YVES GONZALEZ-QUIJANO is a Professor at the Department of Arab Studies at Lumière Lyon2 University and member of GREMMO, Yves Gonzalez-Quijano is the author of d'Arabités numériques. Le printemps du Web arabe (Actes Sud-Sindbad, 2011). 


\section{INTERNET, LE « PRINTEMPS ARABE »ET LA DÉVALUATION DU CYBERACTIVISME ARABE}

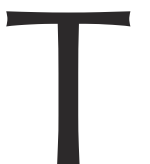

rois ans après leurs débuts, les soulèvements populaires dans le monde arabe n'en finissent pas d'interroger. Après la sidération qui a saisi la plupart des observateurs devant la cadence des changements politiques et leur enchaînement d'un pays à un autre, le temps est désormais aux questions, souvent inquiètes et désabusées, sur le devenir de ces mouvements. Printemps, automne, hiver arabe... : alors que les débats sont loin d'être clos sur le bien-fondé de l'emploi du mot " révolution » pour désigner les transformations dont la région est le théâtre depuis ces trois dernières années, l'opinion, au sens médiatique du terme mais également dans sa dimension académique, a opéré sa propre révolution, un cycle complet qui l'a vu passer de la sympathie à la crainte, voire à l'aversion.

Il en va de même pour un des aspects qui aura, au moins dans les premiers temps, suscité le plus de commentaires, à savoir le rôle joué dans ces événements par les technologies de la communication en général, et par Internet et les réseaux sociaux en particulier. Là encore, les discours, aussi bien médiatiques que savants, sont passés de l'enthousiasme au scepticisme, et même au pessimisme. Après avoir un temps occupé le devant de la scène, les "jeunes rebelles» de la révolution, armés de leurs tablettes numériques, ont laissé la place à d'autres combattants, ceux-là même auxquels l'histoire de la région nous a habitués depuis des décennies : d'un côté, militaires et forces de répression en tous genres, de l'autre, militants et miliciens " de tous poils», au sens propre de l'expression puisque "djihadistes » et «takfiristes» figurent souvent en premières lignes des affrontements.

A l'évidence, il est aujourd'hui très difficile de se risquer à produire des analyses à propos d'un paysage politique qui n'en finit pas de 
changer, et dont on peut penser qu'il continuera à le faire pendant longtemps encore 1 . On pourrait estimer qu'il en va de même pour ce qui est des usages des nouveaux médias dans la sphère publique, usages dont l'expérimentation sur la scène arabe est loin d'avoir livré tous ses enseignements. Pourtant, dans la temporalité extrêmement accélérée du numérique, les trois années qui se sont écoulées permettent de dresser l'esquisse d'un bilan d'ensemble, même si les expériences sont naturellement à la fois variées et contrastées. En revenant sur les séquences ouvertes par le début des soulèvements arabes à la fin de l'année 2010, ce qui suit est ainsi une sorte de "retour sur expérience », une tentative d'évaluation critique du discours que les spécialistes en sciences sociales, et donc en partie l'auteur de ces lignes, ont pu tenir sur le rôle du cyberactivisme dans ces événements réunis sous le nom de «Printemps arabe».

\section{L'INVENTION DU JEUNE REBELLE NUMÉRIQUE}

Avec le recul du temps, la construction d'un étonnant récit sur le rôle des nouveaux médias reste un des aspects remarquables de la première phase des soulèvements arabes - phase qui n'a duré, pour nombre d'observateurs, Marc Lynch² par exemple, que l'espace de quelques semaines, jusqu'à la mi-mars 2011. Du jour au lendemain ou presque, les militants arabes sur Internet sont alors apparus en pleine lumière pour intervenir dans un narrative indéfiniment repris qui leur prêtait un rôle de premier plan dans le succès, assez imprévisible, des manifestations qui ont abouti, très vite, à la chute de Ben Ali en Tunisie et de Moubarak en Egypte pour s'en tenir aux deux destitutions les plus retentissantes.

Cette découverte de l'activisme en ligne dans le monde arabe succédait à des années d'oubli, témoignage d'une indifférence d'autant plus étonnante que les sciences sociales, partout dans le monde mais tout particulièrement aux USA, témoignaient un intérêt marqué pour les effets politiques de la dissémination des techniques numériques dans le corps social. Et les grands noms de ce courant intellectuel étaient loin d'être des marginaux puisque des chercheurs, des hauts responsables de l'administration publique ou encore des dirigeants des principales sociétés de l'économie du savoir tels qu'Ethan Zuckerman (directeur du laboratoire sur les médias citoyens au MIT), Clay Shirky (journaliste spécialisé enseignant, notamment, à l'Université de new York), Alec Ross (conseiller d'Hilary Clinton pour les questions technologiques) ou encore Jared Cohen (actuel directeur de Google Ideas) figuraient dans

1. Gilbert Achcar, 2013.

2. Marc Lynch, 2012. 
différents palmarès des personnalités influentes tel que celui établi par la revue Time Magazine à la fin de l'année 2011.

Malgré cela, et alors que les soulèvements après les élections iraniennes de 2009 - relayés partout dans le monde à travers Twitter avaient suscité un nombre impressionnant de commentaires et d'études, seul un cercle très réduit de spécialistes du monde arabe s'intéressait à cette forme de contestation politique. Sa vitalité était pourtant facile à observer au vu du succès, en Egypte, de l'appel à grève générale lancé sur les réseaux sociaux, en 6 avril 2008, entre autres exemples, pour certains bien plus précoces ${ }^{3}$. Un aveuglement qu'explique - sans le justifier naturellement - la nature du regard traditionnellement porté sur le monde arabe et plus largement sur le monde musulman, condamnés l'un et l'autre, sans autre forme de procès, à la double peine du fanatisme religieux et de l'arriération technologique.

A la faveur des événements en Tunisie puis en Egypte, sans oublier les autres foyers de protestation qui se multipliaient à l'époque, de la Péninsule arabe (Oman, Bahreïn, Yémen...) à l'extrémité marocaine du Maghreb, on a vu par conséquent surgir la figure inédite du « jeune rebelle arabe " dont le portrait - doté de caractéristiques ambiguës, comme une sorte d'hybride entre les banlieusards occidentaux et les manifestants qui avaient investi les places des grandes capitales arabes - allait orner la couverture du magazine Time au titre de la " personne de l'année 4 ». Un jeune rebelle, produit par une génération qu'on disait pourtant perdue pour la politique, et dont la personnalité composite s'exprimait à travers une panoplie d'outils numériques (tablettes, réseaux sociaux, logiciels de montage, palette graphique...), alliés à une détestation (supposée) des pratiques extrémistes de l'islam.

Une figure pour ainsi dire sans passé car, pour lui en donner un, il aurait fallu retourner d'abord à l'histoire des protestations populaires (ouvrières, nationalistes, ou même islamistes) mais aussi à celle des innombrables témoignages d'un soutien « international » (en réalité surtout occidental) aux régimes les plus répressifs de la région. On se souvient ainsi que, quelques jours encore avant sa fuite, le gouvernement français, par la bouche d'un de ses ministres, vantait encore au président Ben Ali le mérite de son savoir-faire dans le domaine du maintien de l'ordre... Du jour au lendemain, le théâtre politique présentait une scène totalement inédite, habitée par une cohorte d'acteurs surgis de

3. Sur cette archéohistoire de I'activisme en ligne dans le monde arabe, voir Gonzalez-Quijano, 2012, p. 59-89.

4. Time, Person of the Year : the Protester, 14 déc. 2011. Pour l'illustration : <http://img.timeinc.net/time/magazine/archive/covers/2011/1101111226_400.jpg>. 
nulle part - ou, si l'on préfère, produits par l'univers éthéré des flux virtuels. Sur le modèle de Waël Ghoneim, ce modern-day Gandhi qui avait " déclenché » la révolution en créant une liste Facebook à la suite de l'assassinat de Khaled Saïd dans un cybercafé d'Alexandrie en juin 2010, on voyait briller de nouvelles icônes de cette révolution. Sans leaders mais également sans affiliations politiques précises, à l'image des " chanteurs de la révolution», comme le rappeur tunisien El-General, condamnant - non sans courage mais en des termes fort généraux la dictature qui sévissait dans son pays ${ }^{5}$. Sans passé à en croire les récits qui ont fleuri à cette époque, sans structure organisationnelle (au sens traditionnel de l'expression), sans idéologie et sans expérience politique pour la plupart d'entre eux, les militants arabes de l'internet n'en étaient pas moins investis de pouvoirs sans limite puisque ce sont eux qui avaient réussi à faire basculer les plus durables des dictatures, à la faveur de ces révolutions désormais placées sous le signe du numérique tout puissant : révolution Facebook, Twitter, Internet...

\section{LE NARRATIVE DE LA «RÉVOLUTION INTERNET»}

L'entrée en scène des jeunes rebelles du numérique accompagnait une mise en récit totalement inédite pour la région du "Printemps arabe ", désormais appréhendé comme la "première révolution du troisième millénaire ", une révolution témoignant, par son importance, de la capacité du numérique à produire des changements politiques radicaux. Sous le vent de folie qui balayait le monde arabe, avec la chute de deux longues dictatures et les inquiétudes de plus d'un régime dans la région, les scénarios ne s'intéressaient plus aux acteurs politiques traditionnels. Toutes les analyses passées, fondées sur les forces d'opposition organisées ou non en partis, s'appuyant ou non sur des alliances locales, représentant ou non des intérêts économiques, idéologiques, stratégiques, étaient comme suspendues, en tout état de cause reléguées au second plan, au profit de la révolte populaire et de ses meneurs numériques.

"Le peuple veut la chute du régime!", ou bien le départ des militaires, ou encore la justice et la dignité : le slogan qui conférait à cette période toute son unité tant il était repris dans les différents mouvements locaux donnait aux acteurs de la révolution un nom qui contribuait à effacer toute structuration politique sous l'utopie d'une

5. Conjointement aux théoriciens du changement politique par internet, ce type d'acteurs, Ghoneim, El-General et d'autres encore, ont eux également les honneurs des palmarès et autres prix internationaux saluant les personnalités marquantes de l'année 2011. 
volonté collective unifiée par la grâce d'un mot d'ordre. Le "peuple » devait pourtant s'organiser pour triompher des forces de répression et pour, ensuite, donner une existence concrète à ses revendications, ce que rendait possible la magie du numérique : là où des années, des décennies de lutte et de protestations s'étaient montrées impuissantes à renverser la moindre dictature, dans aucun des pays de la région, elle permettait enfin, d'abord d'inverser le cours des choses, presque sans effusion de sang, et ensuite de jeter les bases d'une véritable gestion citoyenne participative.

Un tel coup de théâtre était donc rendu possible par l'irruption dans le cours de l'histoire d'un élément inédit, le numérique en général et les "nouveaux médias » en particulier. Pas de place en effet, ou très peu, dans la version standard du narrative dominant pour l'ancien monde, avec ses "vieux médias », en dépit de tous les commentaires passés sur les conséquences de la multiplication des chaînes satellitaires arabes. Oubliée Al-Jazeera, capable de mobiliser les masses par une rhétorique aux accents panarabes ou même panislamiques! Assénée à coups d'éditoriaux dans des quotidiens aussi prestigieux que le New York Times, la vérité des soulèvements arabes, c'était l'avènement d'une "révolution Facebook », avec pour véritable leader Marc Zuckerberg ${ }^{6}$. Le "Printemps arabe » était numérique et illustrait, de la manière la plus éclatante possible, la pertinence des modèles des théoriciens cyberoptimistes.

A quelques nuances près, ceux-ci proposaient un même schéma d'analyse fondé sur une doxa technophile reposant, selon la critique qu'en propose Evgeny Morozov ${ }^{7}$, sur un double credo: d'abord, celui du "solutionisme ", fondé sur la capacités des technologies, et en premier lieu dans le domaine de la communication, à optimiser les problématiques sociales; ensuite, celui de I' «Internet-centrisme, postulant que le Réseau des réseaux, par ses qualités intrinsèques (transparence, décentralisation, ouverture, innovation constante...), était porteur d'une révolution non seulement sociale, ou encore cognitive pour reprendre I'expression de Michel Serres ${ }^{8}$, mais également politique.

Partageant l'illusion qu'internet leur ouvrait une fenêtre donnant directement accès aux réalités du monde arabe ${ }^{9}$, et donc sans s'attarder à analyser la spécificité $d^{\prime}$ 'un contexte social et politique totalement
6. Cohen, 25 janvier 2011.
7. Morozov, 2013.
8. Serres, 2002.
9. Samin, 2012. 
ignoré dans bien des $\operatorname{cas}^{10}$, journalistes, commentateurs de l'actualité et même chercheurs dans bien des cas ont pu ainsi élaborer le canevas commun à la glose cyberoptimiste du "Printemps arabe ». Ils l'ont fait en associant trois éléments réunis dans l'efficace formule d'un manifestant égyptien de l'année 2011 : "Facebook pour planifier les manifestations, Twitter pour les coordonner et YouTube pour le dire au monde. " Les capacités des médiums numériques à démultiplier les moyens de sensibiliser les acteurs potentiels, de les mobiliser, de coordonner de documenter leurs efforts pour gagner des soutiens extérieurs et des protections "expliquaient» comment l'activisme en ligne avait pu réussir là où les formes traditionnelles de l'action politique avaient si longtemps échoué. De fait, durant les premières semaines des soulèvements arabes, tous les pays de la région ou presque étaient gagnés par une même "fièvre ", celle d'une jeunesse qui ne se résignait plus à l'avenir qu'on lui avait était promis et qui réclamait sa part de dignité, de liberté ou simplement d'espoir...

Pourquoi s'attacher au fait que les différentes scènes du « Printemps arabe $" n^{\prime}$ aient pas toujours grand chose en commun, pas davantage du point de vue socio-économique que sur le plan de leur développement numérique? Les mêmes dynamiques devaient régner sur les soulèvements populaires de pays peut-être voisins mais fort éloignés dans les faits, à l'image du Yémen qui, en 2010, possédait un taux d'alphabétisation proche de celui du nombre des utilisateurs d'internet à Bahreïn... L'important, c'était la présence de représentants de la jeunesse en première ligne des manifestations, lesquelles mettaient en évidence nombre d'activistes maniant toute la gamme des réseaux sociaux ; et également le fait que ces derniers - via les médias classiques qui s'en étaient immédiatement emparés - relient les jeunes rebelles arabes entre eux mais également avec le reste du monde. Magie $d^{\prime} u n$ enchaînement logique imparable qui valait confirmation pour ceux qui tablaient, d'une voix de plus en plus affirmée, sur la capacité des ressources numériques à transformer en profondeur les règles du jeu politique, en particulier dans le monde arabe et musulman ${ }^{11}$.

10. On pense ainsi aux déclarations d`Eric Schmidt, P-DG de Google, affirmant avoir découvert, à son retour de Libye, le rôle libérateur d`Internet dans un pays soumis à la censure ! (Cf. Gonzalez-Quijano, 2012, p. 98). II est vrai que les révélations d`Edward Snowden ne viendraient que deux années plus tard!

11. A la veille des soulèvements arabes, Philip N. Howard publiait ainsi The Digital Origins of Dictatorship and Democracy, 2010. 


\section{PREMIÈRES OMBRES AU TABLEAU : DES RÉSEAUX PAS TRÈS NET-S !}

A quel moment l'image enchantée du " Printemps arabe » a-t-elle commencé à perdre de ses couleurs les plus brillantes ? Si le reflux est avéré dès la mi-mars 2011 à en croire un analyste tel que Marc Lynch ${ }^{12}$, la réponse à cette question varie naturellement selon les lieux et même les personnes. Pour ce qui est du versant numérique des choses, on avancera ici qu'un événement, plus que d'autres, fait date en laissant percevoir une première craquelure dans le vernis recouvrant le tableau idyllique des soulèvements numériques. Même s'il a fortement marqué certains esprits à l'époque, on peut néanmoins considérer qu'il a depuis assez largement disparu de la " mémoire vive » de l'actualité, en dépit de ce qu'il indiquait, déjà, sur les usages pervers des réseaux sociaux dans les soulèvements arabes.

A partir de mars 2011, alors que les autorités syriennes semblaient totalement débordées par ceux qui, partout dans le pays, réclament la fin du système Al-Assad, un blog retenait l'attention des internautes du monde entier. Ouvert par une jeune femme qui militait pour la révolution tout en affichant sa différence sexuelle, A Gay Girl in Damascus dressait la chronique des manifestations, puis de leur brutale répression. L'émotion fut portée à son comble lorsque son auteure, Amina Arraf Abdullah, échappa de justesse à la police secrète, grâce au courage de son père, avant de disparaître dans la clandestinité. En juin, un dernier billet, " mis en ligne par une cousine ", annonçait son arrestation, laquelle suscita immédiatement un énorme courant de sympathie, tant dans les médias internationaux que sur Internet où, entre autres mouvements de solidarité, différentes pages Facebook furent créées de par le monde pour réclamer la libération d'un symbole de la révolution syrienne.

Quelques jours plus tard cependant, la vérité de ce récit apparut en pleine lumière, à la suite de différentes enquêtes. Le véritable auteur de ce journal militant n'était en aucun cas une femme, et pas même un Syrien ; il s'agissait d'un nord-américain d'une quarantaine d'années, vaguement étudiant à Édimbourg. Démasqué, il avoua la supercherie et présenta de médiocres excuses à ceux qui s'étaient mobilisés pour Amina, prétextant malgré tout du caractère "plus vrai que nature", et donc en quelque sorte "utile", de sa fiction. Vite recouverte par le flux incessant des informations, et notamment celles, toujours plus sinistres, en provenance de Syrie, I'histoire de la fausse Amina apparaît, rétrospectivement, comme bien plus qu'un simple incident

12. Lynch, 2012. 
dans l'histoire de l'activisme en ligne dans le monde arabe. D'autant plus qu'il n'est pas totalement isolé. Moins médiatisée, la présence, sur les réseaux sociaux d'une journaliste inventée de toutes pièces et intervenant sous le nom de Liliane Khalil (ou encore Habiba Dalal ou Gisèle Nasr) a également été dénoncée quelques mois plus tard. Cette fois-ci, la créature numérique n'était pas dans le camp des protestataires mais dans celui des autorités, celles du Bahreïn en l'occurrence ${ }^{13}$.

Amina et Liliane, deux supercheries jouant subtilement des ressorts bien connus d'un certain "néo-orientalisme» comme le notaient certains commentaires ${ }^{14}$ mais qui étaient surtout les premières manifestations de manipulations numériques incontestables. Avec le temps, celles-ci allaient devenir de plus en plus visibles, qu'il s'agisse des interventions des grandes sociétés Internet pour favoriser (ou non) telle ou telle partie, ou encore de l'appropriation par les différentes forces en conflit, "légitimes » ou "révolutionnaires", des techniques de la guerre numérique. Le Web n'était plus - s'il l'avait jamais été - un univers édénique où $\mathrm{n}^{\prime}$ agissaient que des acteurs oeuvrant en pleine lumière pour faire triompher le bien et la justice. Comme on allait le constater de plus en plus tout au long des divers soulèvements arabes, la Toile avait aussi des zones $d^{\prime}$ ombre ${ }^{15}$. Largement ignorées par les visionnaires de la "révolution numérique » dont les théories reposaient largement sur la croyance en la nature par essence "bonne» des réseaux sociaux ${ }^{16}$, ces replis obscurs recelaient des enjeux qui n'avaient pas toujours échappé à des acteurs directement engagés sur le terrain des luttes concrètes. Animés de toutes sortes d'intentions, bonnes ou mauvaises, ceux-ci en profitaient pour détourner à leur profit les énergies mobilisatrices captées, localement ou internationalement, au sein des réseaux sociaux.

Ce constat, que les faits allaient confirmer toujours davantage, dans le cadre syrien comme ailleurs, il était pourtant très difficile de s'en faire l'écho (et il reste aujourd'hui encore totalement inacceptable pour quelques irréductibles fidèles à la foi numérique). Impossibles à entendre lorsque l'enthousiasme pour les soulèvements arabes se traduisait par toutes sortes d'articles vantant les mérites de cette révolution portée par les réseaux sociaux, ces questions sont néanmoins devenues davantage audibles avec les premières désillusions sur les

13. Kareem, 2011 ; Jones, 2012.

14. Abbas et Boundaoui, 2011.

15. Voir par exemple l'enquête, très documentée, sur la manipulation à travers les réseaux sociaux des circonstances de la mort de Mohamed Bouazizi : Ch. Ayad, 2011.

16. Don't be evil!, tel est le mot d'ordre que s`est donné, depuis ses débuts, la société Google. 
victoires du "Printemps arabe». Pourtant, les mises en garde ne manquaient pas, chez un cyberpessimiste tel qu'Evgueny Morozov ${ }^{17}$, mais également chez certains des acteurs les mieux informés des luttes numériques dans le contexte arabe. Publiée quelques semaines avant le déclenchement des premières protestations en Tunisie, la tribune d'un militant de la première heure, Sami Bengharbia, n'avait pas été assez entendue, en dépit de l'intense débat qu'elle avait provoqué, surtout chez les thuriféraires nord-américains des nouvelles technologies de libération. Dans un texte prémonitoire ${ }^{18}$, le co-fondateur d'une plateforme de l'opposition tunisienne, le site Nawaat, soulignait pourtant les "fausses promesses » $d^{\prime}$ une lutte numérique de plus en plus " polluée » et même mise en danger par les interventions d'acteurs extérieurs, y compris lorsqu'ils étaient animés des meilleures intentions du monde

\section{LE TEMPS DES DÉSILLUSIONS}

Depuis juin 2013, les révélations d'Edward Snowden sont venues s'ajouter aux trop nombreux témoignages, dans le monde arabe, mettant au jour les multiples manières de détourner, au profit de tel ou tel calcul politique, les infinies ressources d'Internet. Sous cet éclairage, et sur fond de défiance par rapport à I' « Occident » et à ses inventions, les réseaux sociaux, vantés autrefois comme les vecteurs idéal de la libération des peuples, sont désormais bien souvent accusés par une partie de l'opinion arabe de participer à une gigantesque " conspiration » qui aurait dupé les énergies protestataires pour dessiner de nouvelles frontières nationales capables de renforcer plus encore la domination des grandes puissances étrangères ${ }^{19}$. Les désillusions des lendemains qui déchantent ont ainsi douché l'incontestable angélisme de nombreux militants en ligne.

D'autant plus que, de leur côté, les régimes locaux s'organisent. Sans doute en partie débordés dans en premier temps par l'utilisation politique des outils numériques et par celle des réseaux sociaux en particulier - même si la Tunisie et l'Egypte figuraient précisément parmi les Etats arabes qui avaient spécifiquement modernisé leurs méthodes de répression pour les adapter aux nouvelles technologie -, ces régimes ont depuis considérablement affiné leurs méthodes. "A la faveur » de l'expérience gagnée lors des divers soulèvements si on peut l'écrire ainsi, la plupart des Etats, à commencer par ceux du Golfe, n'ont cessé $d^{\prime}$ investir dans diverses technologies de surveillance et de mettre en

17. Morozov, 2011.

18. Ben Gharbia, 2010.

19. Parmi de très nombreux exemples, voir notamment Zalloum, 2013. 
place des législations toujours plus strictes à l'encontre des militants de l'internet, eux-mêmes soumis à des sanctions plus sévères ${ }^{20}$.

On aurait tort de croire cependant que cette remise en cause du cyberactivisme, sans doute plus importante encore dans le monde arabe qu'ailleurs, est uniquement le fait de facteurs extérieurs, en raison de l'intrusion de méthodes étrangères aux traditions d'un champ où, selon la devise de Google (Don't be evil!), il n'est pas question " d'être méchant». En effet, les désillusions vis-à-vis du pouvoir des outils numériques ne sont pas seulement liées à la découverte des nombreuses possibilités d'un maniement pervers de leur potentiel politique. En réalité, la dévaluation du cyberactivisme tient tout autant à d'autres facteurs. Moins souvent débattus, ils portent toutefois en eux une dimension critique plus décisive car ce qu'ils remettent en cause, ce n'est plus seulement le dévoiement accidentel d'un système par des acteurs sans scrupules, venus s'immiscer plus ou moins clandestinement dans le domaine des luttes en ligne, mais, plus fondamentalement, le fonctionnement même de ce système, ou plus exactement les vertus politiques qu'on lui a prêtées.

Partielle sans doute, dans la mesure où elle ne touche qu'un des aspects du credo cyberoptimiste, la première de ces désillusions est liée, chronologiquement, aux moments qui ont immédiatement suivi les différents épisodes insurrectionnels. Quels que soient les lieux et les contextes, il est ainsi apparu que les formes d'action associées, d'une manière ou d'une autre, au numérique résistaient peu, ou mal, à un retour à la compétition politique traditionnelle. Que celle-ci se passe dans un cadre démocratique ou non, à l'occasion d'une consultation électorale ou bien au prix d'affrontements armés, le capital acquis, grâce à leurs luttes, par les militants de l'internet s'évaporait aussi soudainement qu'il avait été révélé : quand ils s'étaient vus confier des responsabilités, l'expérience avait rapidement tourné court (le blogueur Slim Amanou devenu un éphémère ministre tunisien de la Jeunesse par exemple); lorsqu'ils s'étaient présentés aux élections, ils avaient été balayés par les partis politiques traditionnels. Avec le temps et le vieillissement de la première génération militante, la blogosphère arabe, avec ses aspects individualistes sinon narcissiques, a révélé combien elle était hiérarchisée par une sorte d'économie de la réputation plus efficace en définitive dans le temps de la contestation que dans celui de la structuration ${ }^{21}$. Même une des rares réussites de la phase post-

20. Sur I>Arabie saoudite, où se met en place une nouvelle instance officielle pour la surveillance des médias sociaux, voir Human Right Watch, 2013.

21. De Angelis et della Ratta, 2014. 
révolutionnaire, l'énorme mobilisation de juin 2013, largement relayé sur les réseaux sociaux par le mouvement Tamarrod en Egypte, devait révéler par la suite qu'elle était en réalité une manipulation du futur pouvoir ${ }^{22}$. A la vérité, elle ouvrait la voie à une restauration militaire et à une répression dont les activistes du Net feraient, le moment venu, eux-mêmes les frais, à l'image de Alaa Abdel-Fattah, militant de la première heure du soulèvement de la jeunesse égyptienne, arrêté en novembre 2013.

D'une manière générale, les mois et même les années qui ont suivi les soulèvements du début de l'année 2011 ont ainsi contribué à faire perdre à l'espace politique numérique une partie du crédit qu'on lui avait prêté. Parce que, dans l'euphorie des premières victoires, on avait sans aucun doute cédé à l'hyperbole pour ce qui est des avantages des luttes en ligne, mais également parce que celles-ci, débarrassées de l'aura révolutionnaire que leur avait conférée le courage des premiers militants du cyberespace, entraient au contraire dans une banalité qui leur ôtait tout caractère d'exception. Au lendemain des révolutions, réussies ou non, et tandis que le nombre des utilisateurs des réseaux sociaux augmentait de façon encore plus vertigineuse que précédemment, l'usage d'Internet et des réseaux sociaux (y compris Twitter, encore modérément présent à l'époque) était totalement récupéré dans la communication politique, y compris par les institutions réputées les plus conservatrices, telles que le ministère des Affaires étrangères en Syrie ou le Conseil suprême des forces armées en Egypte ${ }^{23}$, ainsi qu'au sein de la myriade d'acteurs apparus ou non à la faveur des événements. Avec pour conséquence, naturellement, une sorte de banalisation, et même de dépréciation, comparable au phénomène, bien connu par les spécialistes du domaine, de "bruit informatique » responsable de la perte d'une grande partie de la qualité de l'information.

La dépréciation, de par leur banalisation, des ressources numériques est également associée aux cas, de plus en plus nombreux, de falsification des données dans la communication politique. Bien entendu, le phénomène est depuis toujours ou presque largement pratiqué dans une région où, même les médias réputés sérieux tels que Al-Jazeera ne sauraient échapper à une instrumentalisation qui, dans le cas de la chaîne qatarie, est apparue de plus en plus manifeste au fil des mois qui ont suivi les soulèvements du début 2011. Source de nombreuses interrogations, la nature de la couverture médiatique des événements par les grands médias régionaux (en Libye ou a contrario

22. Iskandar, 2013.

23. Voir El-Khalili, 2013. 
au Bahreïn pour s'en tenir à ces seuls exemples) a de plus en plus associé dans ses dérives les ressources numériques devenues l'ordinaire des grandes chaînes satellitaires. Ouverte en Egypte au temps de la chute de Moubarak, la programmation de la chaîne Al-Jazeera Direct (Al-Jazeera mubâshir) a pu être ainsi très largement constituée de documents récupérés sur Internet, mais le phénomène s'est prolongé, avec des pratiques journalistiques nettement plus critiquables, à partir du moment où la première chaîne d'information dans le monde arabe, talonnée par sa rivale la saoudienne Al-Arabiyya, a pris fait et cause pour les rebelles syriens (fin mars 2011).

Dans I'univers d'Internet, on peut estimer que cette réalité était restée longtemps marginale, ne serait-ce parce que la stratégie des blogueurs et autres journalistes-citoyens, les premiers, historiquement, à avoir investi ce champ, consistait précisément à tirer profit en visibilité et en crédibilité des criantes lacunes du système $d^{\prime}$ 'information. Pourtant, le réel capital de sympathie dont disposaient les médias en ligne auprès d'une partie non négligeable du public arabe a rapidement été dilapidé, surtout là où le manque d'expérience s'est traduit par une faible structuration des tribunes militantes, devenues par ailleurs de plus en plus inaudibles au sein de la cacophonie des sources discordantes nouvellement apparues ${ }^{24}$.

\section{LA PERTE DE LA VIRGINITÉ POLITIQUE}

Après avoir abandonné sa singularité, c'est en quelque sorte également sa "virginité politique » que la parole militante numérique a perdu à la suite de ce que l'on a appelé le "Printemps arabe», en particulier dans le conflit syrien qui, par le caractère dramatique de ses affrontements, pourrait bien avoir contribué à ruiner, définitivement, toute croyance sincère (ou si l'on préfère non naïve) dans les vertus fondamentalement démocratiques de l'activisme politique en ligne. Avec la démocratisation de l'internet arabe depuis si longtemps annoncée $^{25}$ et, par conséquent l'intensification des échanges numériques qu'elle a produite et qui s'est accélérée sur les réseaux sociaux depuis les divers soulèvements arabes, on a pu remarquer, en plus d'un lieu, la «polarisation» des acteurs poussés, par la dynamique propre aux interactions numériques, à se retrancher dans leur environnement politique immédiat plutôt qu'à ouvrir des espaces de dialogue et d'échange ${ }^{26}$. Dans des contextes particulièrement tendus

24. Pour le cas syrien, voir De Angelis, 2011.

25. Gonzalez-Quijano, 2003.

26. Lynch, 2013. 
- en Syrie notamment à partir du moment où le soulèvement pacifique d'une partie de la population syrienne a évolué vers des violences de plus en plus interconfessionnelles attisées par les intérêts géopolitiques de puissances régionales et internationales -, il est apparu que les réseaux sociaux offraient un terreau fertile au développement des pires violences sectaires. On ne pense pas nécessairement ici aux délires des factions les plus extrémistes, celles des défenseurs les plus acharnés d'un régime dont la nature est connue depuis des années ou celles qui accueillent des combattants étrangers venus combattre au nom de ce qu'ils interprètent comme l'idéal révolutionnaire islamiste, mais au contraire aux internautes syriens ordinaires, pris dans la tourmente qui s'est abattue sur leur pays.

Pour ceux-là, quel que soit leur camp et même si leur engagement sur la scène politique ne prend que des formes modestes, les réseaux sociaux sont devenus de véritables machines à créer de la violence, ou encore une arme à part entière dont l'acquisition et l'utilisation finit par altérer la stratégie des acteurs qui dictent leur comportement, sur le terrain ou sur la Toile, en fonction de cette nouvelle réalité aux effets déformants ${ }^{27}$. Contrairement à ce qu'avaient imaginé la plupart de ceux qui se sont efforcés de réfléchir à ses conséquences politiques, on constate ainsi dans l'épouvantable conflit syrien que l'essor $d^{\prime}$ Internet ne favorise nullement la constitution d'une sphère publique de substitution particulièrement utile à la réduction de l'autoritarisme. II ne favorise pas non plus le poids de médiateurs générant par exemple des échanges de pairs à pairs, construisant, pierre à pierre (sur un mode incrémental dans le jargon des spécialistes) une agora numérique dont la dynamique ne peut manquer de rejaillir dans le jeu politique. Depuis que la violence a commencé à se déchaîner sur la population syrienne, les réseaux sociaux et plus largement tout l'espace numérique n'apaisent rien, n'ouvrent à aucun débat, ne dessinent aucun avenir négocié, dénouent, un à un, tous les liens qui tissent la toile sociétale. A grand renfort d'images plus glaçantes les unes que les autres, de vidéos invérifiables qu'on affiche comme autant de preuves justifiant des mises à l'index et des "listes de la honte » (qâ'imat al-'âr) qui valent, dans un camp comme dans l'autre, ordre d'exécution, l'espace numérique relaie et cristallise le pire des terreurs et des haines identitaires.

De par leur destinée inévitablement difficile et par moments dramatique, les révolutions arabes ne sont pas ce laboratoire des révolutions du troisième millénaire auquel on a trop vite voulu croire. Pas plus que les médias de masse, comme on a pu le dire il y a plus d'un

27. Harkin, 2013. 
demi-siècle maintenant ${ }^{28}$, les médias sociaux ne sont porteurs d'une révolution sociale et politique. En tout cas pas de cette manière-là. $C^{\prime}$ est dans une autre temporalité que les outils numériques provoquent des effets que I'on peut qualifier de révolutionnaires. On peut ainsi considérer que leur extraordinaire essor auprès de la jeunesse arabe signe, tôt ou tard, la fin d'un cycle dans l'histoire de la région, celle d'une utopie politique, celle d'une "idée arabe " apparue avec la révolution de la communication que fut, pour la région, l'introduction de l'imprimerie au cours du XIXe siècle. On peut aussi espérer que la fin de cette première Renaissance (Nahda) ouvre à d'autres propositions politiques, en relais des " arabités numériques » qui se nourrissent des modalités actuelles des flux communicationnels ${ }^{29}$.

28. Lerner, 1958.

29. Sur ce point, voir le dernier chapitre et la conclusion de Arabités numériques. Le printemps du Web arabe, op.cit. 


\section{BIBLIOGRAPHIE}

Abbas, A.; Boundaoul, A., 2011, "The Politics Behind the Roleplay ", Kabobfest.org: <http://www.kabobfest.com/2011/06/a-gay-girl-indamascus.html $>$.

AchAR, G., 2013, Le peuple veut. Une exploration radicale du soulèvement arabe, Sindbad, Paris.

AyAD, C., 2011, "La révolution de la gifle », Libération, <http://www. liberation.fr/monde/2011/06/11/la-revolution-de-la-gifle_741980>

Ben GHarbia, S., 2010, "The Internet Freedom Fallacy and the Arab Digital Activism », Nawaat: <http://nawaat.org/portail/2010/09/17/ the-internet-freedom-fallacy-and-the-arab-digital-activism/>.

COHEN, R., 2011, "Facebook and Arab dignity ", The New York Times: <http://www.nytimes.com/2011/01/25/opinion/25iht-edcohen25. html>.

De angelis, E., 2011, « The State of Desarray of a Networked Revolution. The Syrian's Uprising Information Environment », Sociologica, 3.

De angelis, E.; DELLA RATTA, D, 2014, " Mind the Gap: Bridging Knowledge and Practices of Activism at the Forth Arab Meeting ", Jadaliyya: <http://www.jadaliyya.com/pages/index/18040/mindthe-gap_bridging-knowledge-and-practices-of-an>.

EL-KHALILI, S., 2013, "Social media as a government propaganda tool in post-revolutionary Egypt », First Monday, Vol. 18, nº 3.

Gonzalez-QuiJANo, Y., 2012, Gonzalez-Quijano (Yves), Arabités numériques. Le printemps du Web arabe, Sindbad, Paris.

GonZALEZ-QUiJANO, Y., 2003, "A la recherche d'un Internet arabe: démocratisation numérique ou démocratisation du numérique?", Maghreb-Machrek, $\quad \mathrm{n}^{\circ} 178$ : <http://halshs.archives-ouvertes. fr/view_by_stamp.php? \&halsid=i0c5mtrsc $81 / 7$ q 1 fuci $041 \mathrm{cr}$ $70 \&$ label=SHS\&langue $=$ fr \&action_todo $=$ view \&id=halshs $00646377 \&$ version $=1>$.

Harkin, J., 2013, "Good media, bad politics? New media and the Syrian conflict», Reuters Institute Fellowship Paper, University of Oxford: <http://reutersinstitute.politics.ox.ac.uk/sites/default/files/ Good\%20media\%2C\%20bad\%20politics\%20New\%20media\%20 and $\% 20$ the $\% 20$ Syrian $\% 20$ conflict.pdf $>$.

HowarD, P. N., 2010, The Digital Origins of Dictatorship and Democracy. Information Technology and Political Islam, Oxford University Press, Oxford.

Human RIGHTS WATCH, 2013, Challenging the Red Lines: Stories of Right Activists in Saudi Arabia.

ISKANDAR, A., 2013, "Tamarod: Egypt's Revolution Hones its Skils», Jadaliyya: <http://www.jadaliyya.com/pages/index/12516/tamarod_ egypts-revolution-hones-its-skills>. 
JONEs, M. O., 2012, "Hoax Journalist Lilane Khalil Returns, This Time as Habiba Dalal ", Marc Owen Blog : <https://marcowenjones.wordpress.com/2012/01/29/the-return-of-liliane-khalil/>

KAREeM, M., 2011, "Bahreïn : Liliane Khalil, une nouvelle imposture ou une opération de propagande? », Global Voices : <https:// fr.globalvoicesonline.org/2011/08/08/75759/>.

Lerner, D., 1958, The Passing of Traditional Society. Modernizing the Middle East, Free Press, New York.

LyNCH, M., 2012, "When the Empire Struck Back», Foreign Policy: <http://foreignpolicy.com/2014/09/11/the-empire-strikes-back-3/>

LyNCH, M., 2012, The Arab Uprising: The Unfinished Revolutions of the Middle East, Public Affairs, New York.

LYNCH, M., 2013, "Twitter Devolution. How social media is hurting the Arab Spring 》, Foreign Policy: <http://www.foreignpolicy.com/articles/2013/02/07/twitter_devolutions_arab_spring_social_media>.

Morozov, E., 2011, The Net Delusion, The Dark Side of the Internet Freedom, Allen Lane, Londres.

Morozov, E., 2013, To Save Everything Click Here. Technology, Solutionism and the Urge to Fix Problems That Don't Exist, Allen Lane, Londres.

OWEN JONES, M., 2012, "Hoax Journalist Lilane Khalil Returns, This Time as Habiba Dalal », Marc Owen Blog: <http://marcowenjones. wordpress.).com/2012/01/29/the-return-of-liliane-khalil/>.

SAmin, N., 2012, "Saudi Arabia, Egypt and the Social Media Moment ", Arab Media \& Society, $\mathrm{n}^{\circ}$ 15: < http://www.arabmediasociety. $\mathrm{com} /$ ?article $=785>$.

Serres, M., 2002, "Les nouvelles technologies »,Tr@nsit online, $\mathrm{n}^{\circ} 22$ : $<$ http://www.iwm.at/read-listen-watch/transit-online/les-nouvellestechnologies/>.

Zalloum, A, 2013, Al-rabî' al-'arabi, thawra am fawda (ghayr) khallaqa [Le printemps arabe, révolution ou chaos (non)constructeur], Beyrouth, Al-mu'assassa al-'arabiyya lil-dirâsât wal-nashr. 